\title{
EXPERIMENTAL STUDY OF BEAT WAVE EXCITATION OF HIGH PHASE VELOCITY SPACE CHARGE WAVES IN A PLASMA FOR PARTICLE ACCELERATION
}

\author{
C. Joshi, C. E. Clayton, C. Darrow, and D. Umstadter \\ University of California, Los Angeles CA 90024
}

\section{ABSTRACT}

In this paper we present the first detailed study of the excitation of high phase velocity plasma waves by colinear optical mixing. The plasma wave frequency and wavenumber, hence the phase velocity, are directly measured using Thomson scattering. The amplitude of the plasma wave and therefore the longitudinal electric field is inferred from the magnitude of the Thomson scattered light. For a modest laser intensity of $\sim 1013 \mathrm{~W} / \mathrm{cm}^{2}$ in each frequency, the excited space-charge plasma wave has measured longitudinal electric fields of the order $1 \mathrm{GeV} / \mathrm{m}$, in reasonable agreement with the fluid theory.

\section{INTRODUCTION}

Recently there has been a considerable amount of interest in exploring whether longitudinal electric field associated with spacecharge plasma density waves propagating close to the speed of light can be used to accelerate particles to very high energies in a very short distancel. The motivation for this approach comes from the fact that the maximum electric field associated with such a wave scales as $\sqrt{\mathrm{n}_{\mathrm{e}}}(\mathrm{cm}-3)$ Volts $/ \mathrm{cm}$. Thus for a plasma density of $10^{16}$ electrons $/ \mathrm{cm}^{3}$ the maximum electric field is $10 \mathrm{GeV} / \mathrm{m}$, orders of magnitude greater than the accelerating gradients used in the current accelerators. It has been proposed that such a large amplitude, relativistic (fast) space-charge plasma wave can be driven by beating two lasers $\left(\omega_{0}, k_{0}\right)$ and $\left(\omega_{1}, k_{1}\right)$ in a plasma such that

$$
\begin{aligned}
& \omega_{0}-\omega_{1}=\omega_{p} \\
& \underline{k}_{0}-\underline{k}_{1}=\underline{k}_{p} .
\end{aligned}
$$

Here $\omega_{p}$ is the plasma wave frequency and $\underline{k}_{p}$ is the plasma wave wavenumber. Considerable theoretical work and numerous simulations have been carried out on this scheme. 2,3 However, to-date there has been no conclusive evidence experimentally that such high longitudinal fields can be generated in a plasma by beating two colinearly propagating laser beams. 4 In this paper we report the first detailed study of the excitation and detection of high phase velocity plasma waves by colinear optical mixing; this work was carried out at UCLA as part of the program on the Plasma Beat Wave Accelerator. 
two frequencies, corresponding to wavelengths $9.56 \mu \mathrm{m}$ and $10.59 \mu \mathrm{m}$, is used to excite a beat wave in a hydrogen plasma. Both laser wavelengths, generated from a single mode-locked TEA oscillator are amplified through the same gain medium and are finally focused using a single $\mathrm{ZnSe}, \mathrm{f} / 7.5$ lens to ensure spatial overlap and temporal coincidence. The typical line intensities used, expressed as the normalized parameter $\mathrm{v}_{0} / \mathrm{c}$, where $\mathrm{v}_{\mathrm{o}}(0,1)=\mathrm{eE}_{0,1} / \mathrm{m} \omega_{0,1}$ is the oscillating velocity in the laser field, are $\left(v_{0} / c\right) 10.6 \mu \mathrm{m} \sim 0.03$ and $\left(v_{0} / c\right) 9.6 \mu \mathrm{m} \sim 0.015$. The resonant density where the plasma frequency is equal to the difference frequency between the two lasers is $1.15 \times 1017 \mathrm{~cm}^{-3}$. To produce this density we use a high current arc discharge to create a pre-ionized plasma using $\mathrm{H}_{2}$ as the fill gas and then use the laser beams to accomplish full ionization. For our laser parameters, the plasma wave should grow to a saturated amplitude $\tilde{\mathrm{n}} / \mathrm{n}_{\mathrm{O}} \sim 0.08$ in roughly $500 \mathrm{ps}$ according to fluid theory assuming relativistic saturation. 3 This implies a longitudinal field of $2.8 \mathrm{GeV} / \mathrm{m}$. Since the time to saturation. is relatively long, it is in principle possible to time resolve the growth of the plasma wave, thereby making comparison with theory possible. On the other hand, one must hold the plasma density at a constant value for $1000^{\prime} \mathrm{s} / \omega_{\mathrm{p}}$ which is a very difficult thing to accomplish.

The experimental set-up is shown in Fig. 1. Incident laser parameters are monitored by splitting a fraction of the incident energy using the beamsplitter B1. This beam is split again using the beamsplitter $B 2$ and is then focused onto a calorimeter which monitors the incident pulse energy. The rest of the beam is incident onto a normal incidence $50 \mathrm{\ell} / \mathrm{mm}$ grating which disperses the two wavelengths. Using a concave mirror $M_{1}$ the two wavelengths are then focused onto two separate photon drag detectors which monitor the pulse shape and the relative amplitudes of the $10.59 \mu \mathrm{m}$ and $9.56 \mu \mathrm{m}$ laser lines. The backscattered radiation from the plasma is also sampled using the beamsplitter BI. Since the backscattered radiation generally consists of signal from stimulated Brillouin scattering (SBS) and stimulated Raman scattering (SRS), another beamsplitter $B 3$ is used to split the backscattered radiation. Reflected signal from $B 3$ is sent to a scanning Fabry-Perot interferometer. The output of the F-P interferometer is detected by a Ge:Hg liquid He cooled detector. By scanning the plate separation of the F-P, a spectrum of the Brillouin backscattered light is obtained. Plasma temperature can be deduced from the red-shift of the backscattered light compared to the incident light. The backscattered radiation transmitted by the beamsplitter B 3 is sent through a gas cell containing $\mathrm{SF}_{6}$ and ethanol which strongly absorbs radiation in the vicinity of $10.6 \mu \mathrm{m}$ and $9.6 \mu \mathrm{m}$, respectively. Any Raman scattered light is typically of a wavelength $>11.5 \mu \mathrm{m}$ for our plasma conditions and is therefore transmitted by the gas cell. Infrared bandwidth filters $(\Delta \lambda \sim 2 \mu \mathrm{m})$ are used in conjunction with a $\mathrm{Ge}: \mathrm{Cu}$ cold detector to determine the wavelength range of scattered radiation. The Raman scattered radiation is not intense enough to be dispersed using a grating, so that the scattered 


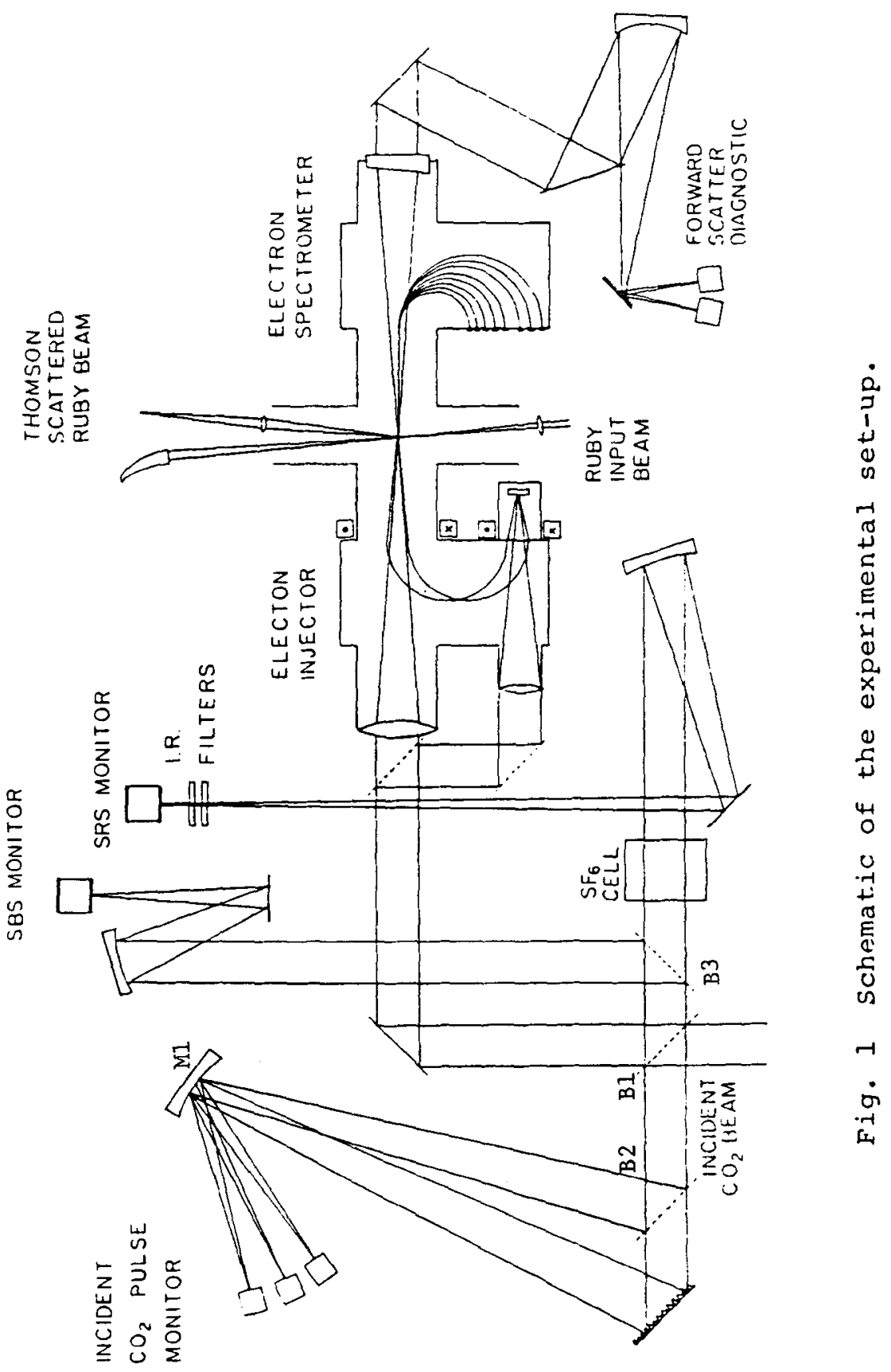


light wavelength and hence the plasma density cannot be measured directly. Instead Ruby laser Thomson scattering is used to detect the frequency of the plasma wave excited during Raman scattering directly. By changing the angle at which the scattered radiation is observed, one can observe plasma and ion modes of different wavenumber.

The $\mathrm{CO}_{2}$ laser beam transmitted by the plasma is collimated, split-off and its wavelength content analyzed using an IR spectrograph. Using a grating and bandpass filters, signals as small as $10^{-8}$ of the incident can be measured. This spectrometer is used to detect the forward Stokes and anti-Stokes radiation generated in the plasma.

\section{OBJECT OF CURRENT EXPERTMENTS}

The object of the current experiments is to quantitatively understand the physics of the beat-wave excitation and competing plasma processes. Some of the physics issues are: (a) Does the frequency difference between the two lasers, $\omega_{0}-\omega_{1}$, exactly have to be $\omega_{\mathrm{p}} ?^{5}$ If not, what is the width of the resonance? To answer this question one must have an extremely well diagnosed (better than 1\%) plasma source. (b) How rapidly does the plasma wave build-up? Particle simulations suggest that the plasma-wave build-up is consistent with the growth rate given by the fluid theory. Ideally the growth time should be on the order of the laser pulse risetime. On the other hand, the build-up should occur faster than any instabilities. (c) What is the amplitude of the plasma wave at saturation and how does it depend upon the laser beam intensities and pulselength? (d) What is the saturation mechanism? Rosenbluth and Liu's model predicts that as the plasma wave grows, the relativistic effect on the frequency mismatch becomes important leading to the saturation of the plasma wave at an amplitude much smaller than that expected at wavebreaking. Their theory, however, still predicts large plasma waves $(0(10 \%))$ for intense laser fields $\left(v_{0} / c \sim 0.1\right)$. An important question is whether other effects that are not included in their model such as collisions, plasma-wave convection or wave-wave coupling saturate the plasma wave to an amplitude that is lower than that due to relativistic mismatch? (e) What are the competing processes and how deleterious are they? Some of the competing processes are stimulated Brillouin scattering, Raman back and side scattering, self-focusing, filamentation and the parametric decay instability. Our current experiments are aimed at addressing these issues.

\section{THOMSON SCATTERING}

The theory for Thomson scattering of a probe laser beam from a single ion or plasma wave is analogous to Bragg scattering of $x$-rays from periodic electron distributions in crystals. In Thomson scattering, as in Bragg scattering, the radiation field of each electron oscillating in the probe beam's electric field is 
calculated and the total field is found by integrating over all the electrons in the scattering volume. In an ion wave both the electron and ion density is modulated at a certain wavenumber, whereas in a plasma wave only the plasma electrons are bunched at a given wavenumber with the ions forming a uniform background. It is found that the scattered intensity is maximized when $\Delta \underline{k}=\underline{k}_{i}-\underline{k}_{s}$ where $\Delta k$ is the wavevector of the ion or plasma wave, $k_{i} \overrightarrow{i s}$ the wavevector of the incident probe wave and $k_{s}$ is the wavevector of the scattered probe wave. The Bragg condition is satisfied when

$$
\Delta \underline{k}=2 k_{i} \sin \left(\theta_{s} / 2\right)
$$

where $\theta_{S}$ is the scatter angle. Since in an ion wave, the phasing of the electron and ion bunches travels at the ion acoustic velocity $\mathrm{vac}$, the scattered light is either frequency up- or down-shifted by $\omega_{a c}=\Delta \mathrm{kv}_{\mathrm{ac}}=2 \mathrm{k}_{\mathrm{o}} \mathrm{vac}_{\mathrm{ac}}$. The phasing of the electron bunches in a plasma wave propagate much faster at a speed $\omega_{\mathrm{p}} / \Delta \mathrm{k}$ and the scattered signal is either frequency up- or down-shifted by $\omega_{p}$.

The scattered light will have a small angular spread about $\theta_{\mathbf{S}}$ because of diffraction. If all the light is collected, the Thomson scattered power $P_{T S}$ is given by

$$
P_{\mathrm{TS}}=\mathrm{p}_{1}\left(\frac{\pi}{2} \frac{\tilde{\mathrm{n}}}{\mathrm{n}_{\mathrm{o}}} \frac{\mathrm{n}_{\mathrm{o}}}{\mathrm{n}_{\mathrm{C} i}} \frac{\mathrm{d}}{\lambda_{\mathrm{i}}}\right)^{2}
$$

where $P_{1}$ is the power of the probe beam intercepted by the ion/plasma wave, $d$ is the thickness of the ion/plasma wave along the probe beam direction, $n_{c i}$ is the critical density of the probe beam and $\lambda_{i}$ is the wavelength of the probe beam.

In order to get a small spread of scattered light about the scattering angle $\theta_{s}$ and hence get a good $k$ resolution, it is necessary to have many periods of the wave in the scattering volume. In an underdense plasma, $\omega_{p} \ll \omega_{o}$, the wavenumbers of the ion wave due to SBS and the plasma wave due to SRS in the backscatter direction are almost the same and $\simeq 2 \mathrm{k}_{\mathrm{o}}$. The scatter angle for both these waves thus conveniently turns out to be $-7.5^{\circ}$ when a ruby laser is used as a probe. Since the $\Delta \mathrm{k}$ is large and the scatter angle is reasonably large, spherical optics can be used to both focus the probe beam so that it covers many wavelengths and collect the scattered light. However, plasma waves produced by Raman forward scattering or colinear optical mixing have a wavelength that is $\left(\omega_{0} / \omega_{p}\right)$ times the incident laser wavelength. For instance for a $10.6 \mu \mathrm{m}$ laser propagating into a $1017 \mathrm{~cm}^{-3} \mathrm{plasma}, \omega_{0}=10 \omega_{\mathrm{p}}$ and the Raman forward plasma wave has a wavelength of $\sim 100 \mu \mathrm{m}$. The scatter angle is therefore only $0.4^{\circ}$. To probe many periods of the plasma wave, therefore, one has to use cylindrical focusing of the probe ruby beam. A combination of cylindrical optics also has to be used to collect the scattered 1ight and physically separate it from the unscattered probe light. A novel technique has been developed to do this and is discussed elsewhere. 6 


\section{PLASMA DIAGNOSTICS}

First, the laser is operated in a single frequency mode $(10.6 \mu \mathrm{m})$ and fired into the pre-ionized arc plasma. The peak intensity of $-1013 \mathrm{~W} / \mathrm{cm}^{2}$ in the focal volume easily exceeds the Brillouin backscatter and Raman backscatter thresholds but does not exceed the Raman forwardscatter threshold. The backscatter diagnostics show that up to $10 \%$ of the incident energy is Brillouin backscattered, whereas, the amount of light being Raman backscattered is typically less than $0.1 \%$ of the incident. The infrared spectrograph in the forward direction failed to detect any downshifted (Stokes) signal greater than $10^{-8}$ of the incident. Therefore, Raman forwardscatter can be neglected compared to backscatter.

In order to detect the SBS driven fon wave and SRS driven plasma wave, the ruby laser scattering is set up for $7 \frac{1}{2}{ }^{\circ}$. In Fig. 2 we can see the Thomson scatter data at this scattering angle for four different shots. On the right hand side of each picture we see a peak corresponding to Thomson scattering from SBS. The frequency of the fon wave as measured using the Fabry-Perot is typically $\sim 10-15 \mathrm{GHz}$. Since the grating dispersion for resolving the Thomson scattered light is $5 \AA / \mathrm{mm}$, any frequency shift less than $10 \mathrm{GHz}$ cannot be resolved and, therefore, the signal essentially appears at the incident ruby wavelength. Nonetheless, from extensive previous measurements we do know that this signal is from an ion wave driven by $\mathrm{SBS}^{7}$.

To the left hand side of the SBS peak, a second peak is seen which varies in position w.r.t. the SBS peak. This peak is characteristic of Raman backscattering and the frequency shift relative to the SBS peak is a direct measure of $\omega_{p}$, and hence, the plasma density. This diagnostic is a very sensitive measure of the plasma density (better than $2 \times 10^{15} \mathrm{~cm}^{-3}$ ). By varying the gas fillpressure, arc-voltage and the relative time between pre-ionization and the main laser, plasmas of different density can be produced as can be seen in Fig. 2. The plasma density can be tuned close to the resonant density of $1.15 \times 1017$ very accurately using this technique.

If the $\mathrm{CO}_{2}$ laser intensity is reduced by $30 \%$ the Raman peak is found to disappear. Thus we can estimate the density scalelength assuming the threshold condition for linear and parabolic density profiles to be

$$
\begin{aligned}
& \mathrm{I}\left(\mathrm{W} / \mathrm{cm}^{2}\right) \geq 4 \times 10^{17 / \lambda_{\mathrm{O}} \mathrm{L}} \quad \text { (1inear) } \\
& \mathrm{I}\left(\mathrm{W} / \mathrm{cm}^{2}\right) \geq\left[5 \times 10^{15} /\left(\lambda_{\mathrm{o}}^{2 / 3} \mathrm{~L}^{4 / 3}\right)\right] \mathrm{T}_{\mathrm{e}}^{1 / 3}\left(\mathrm{n}_{\mathrm{c}} / \mathrm{n}_{\mathrm{o}}\right)^{1 / 3}
\end{aligned}
$$

This gives $200<\mathrm{L}(\mu \mathrm{m})<1000$, assuming $\mathrm{T}_{\mathbf{e}}=50 \mathrm{eV}$. Here

$$
\mathrm{L}=\left[\frac{1}{\mathrm{n}_{\mathrm{o}}}\left(\frac{\mathrm{dn}}{\mathrm{dx}}\right)\right]^{-1} \quad\left(\text { inear) or } \mathrm{L}=\left(\frac{-1}{2 \mathrm{n}_{\mathrm{o}}} \frac{\mathrm{d}^{2} \mathrm{n}}{\mathrm{dx}^{2}}\right)^{1 / 2}\right. \text { (parabolic) }
$$



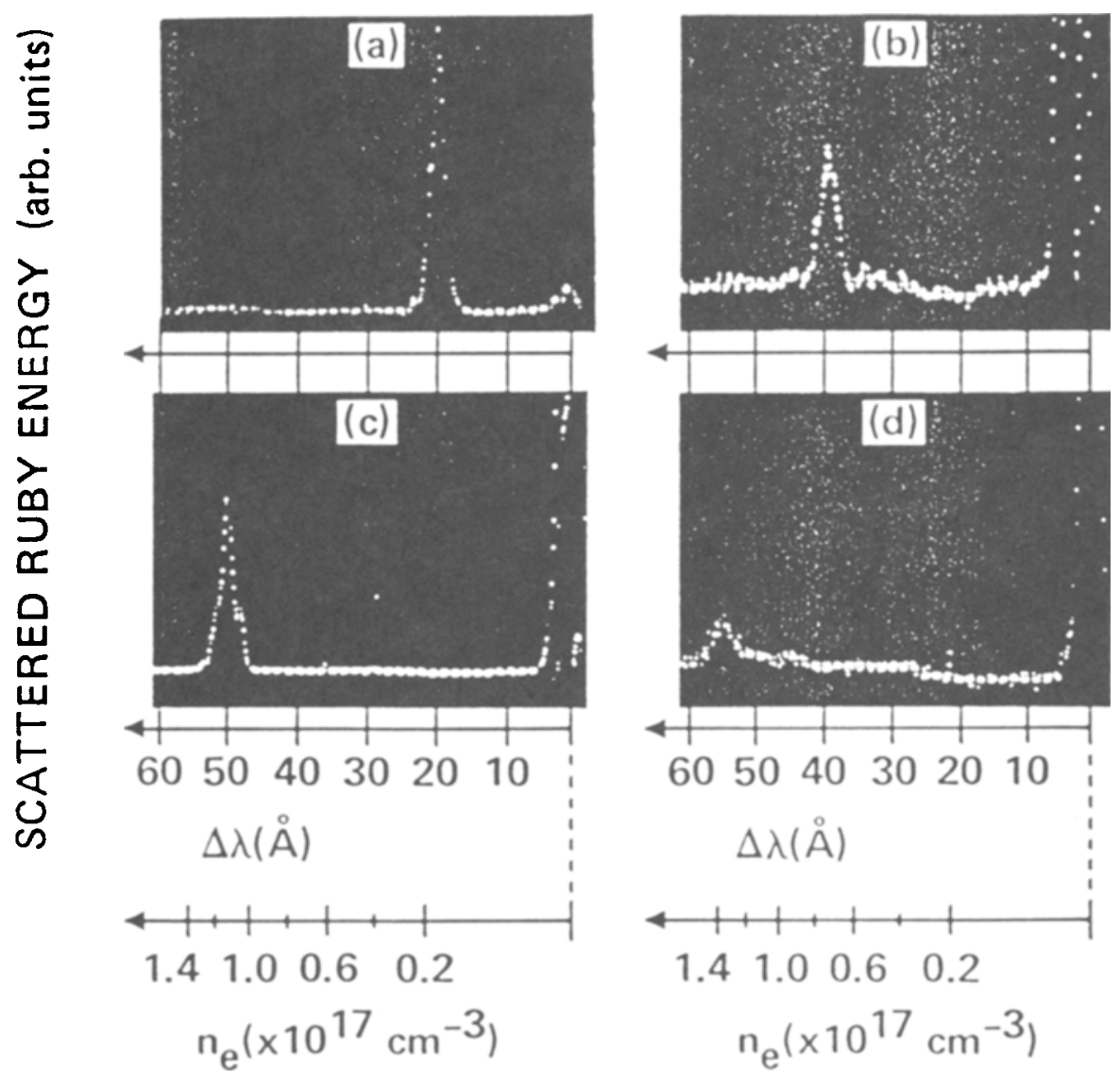

Fig. 2 Thomson scattered light spectra taken at $7 \frac{1}{2}{ }^{\circ}$ for different plasma densities. 
Apart from gross changes in density, the density profile has fine structure due to SBS. By spatially probing the plasma along the depth of focus of the $\mathrm{CO}_{2}$ laser, the ion wave was determined to be approximately $1 \mathrm{~cm}$ in length. Using the Bragg formula and assuming a fully saturated ion wave we obtain a density fluctuation level of $\tilde{\mathrm{n}} / \mathrm{n}_{\mathrm{o}} \sim 2 \%$ which of course has a periodicity of $5 \mu \mathrm{m}$.

\section{RESULTS}

Observation of the "fast" wave: The conclusive evidence for the existance of the high phase velocity ("fast") plasma wave excited by beating of two laser beams resonantly in the plasma comes from ruby laser Thomson scattering. The scattering angle is adjusted to $\mathrm{k}$ match to the fast wave. Once the conditions for obtaining the correct plasma density for beat excitation have been established as discussed previously the plasma is irradiated by two laser wavelengths $(10.6 \mu \mathrm{m}$ and $9.56 \mu \mathrm{m})$ in a single laser pulse. Fig. 3(a) shows the frequency shift of the ruby light from the stray position to be exactly $\Delta \omega=\omega_{p}$. The fast plasma wave is only seen to occur under two frequency illumination and never under single frequency illumination. Thus Raman forward instability can be ruled out as the excitation mechanism. Secondly, whereas the Raman backscatter plasma wave (slow wave) is excited over a range of densities, the beat wave excited fast wave only occurs at $\Delta \omega$ $\omega p$. If the plasma density is tuned off resonance, the scattered signal drops (but does not shift in frequency) rapidly. We do not as yet have a quantitative measure of the width of the resonance. By moving the fiber optic which collects the scattered light on a shot-to-shot basis, the $k$ spectrum of the fast wave has been obtained. It is shown in Fig. 3(b). In spite of the large shotto-shot variation in the scattered light, it is clear from this figure that the scattered light spectrum is peaked about $k_{p}=$ $k_{0}-k_{1}$. These direct measurements of the plasma wave frequency and the wavenumber have enabled us to unambiguously identify the wave as being the fast plasma wave $(\omega / \mathrm{k} \sim c)$. Amplitude of the fast wave: The ruby laser pulse used for Thomson scattering is $25 \mathrm{~ns}$ (FWHM) long and its spatial extent is $1 \mathrm{~cm}$. The fraction of the ruby power intercepted by the fast wave (and therefore available for scattering) is

$$
P_{i}=P_{o}\left(\frac{\tau_{f}}{\tau_{o}}\right)\left(\frac{L_{f}}{L_{o}}\right)
$$

where $P_{O}, \tau_{0}$, and $L_{0}$ are the total incident ruby power, pulselength and spatial extent, respectively, and $\tau_{f}$ and $L_{f}$ are the duration and the spatial extent of the fast wave, respectively.

The spatial profile of the fast wave was constructed by placing a $500 \mu \mathrm{m}$ mask in the input ruby beam such that only about 5 wavelengths of the plasma wave could scatter the ruby light on a given shot. The data is shown in Fig. 4. The fast plasma wave was found to be roughly $1.8 \mathrm{~mm}$ (or -18 wavelengths) long. 


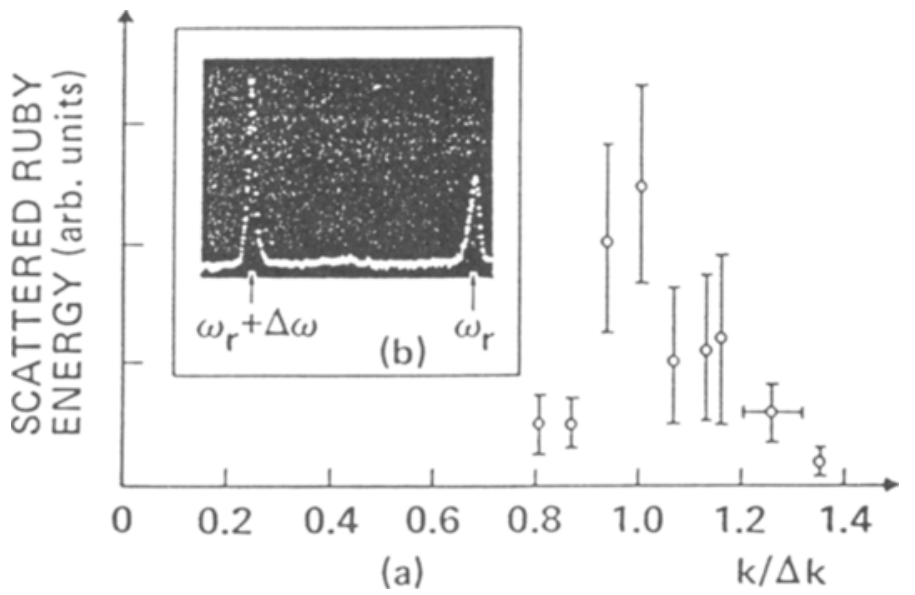

Fig. 3 (a) k spectrum of the fast wave and (b) Thomson scattering at $0.4^{\circ}$ showing the resonant frequency shift.

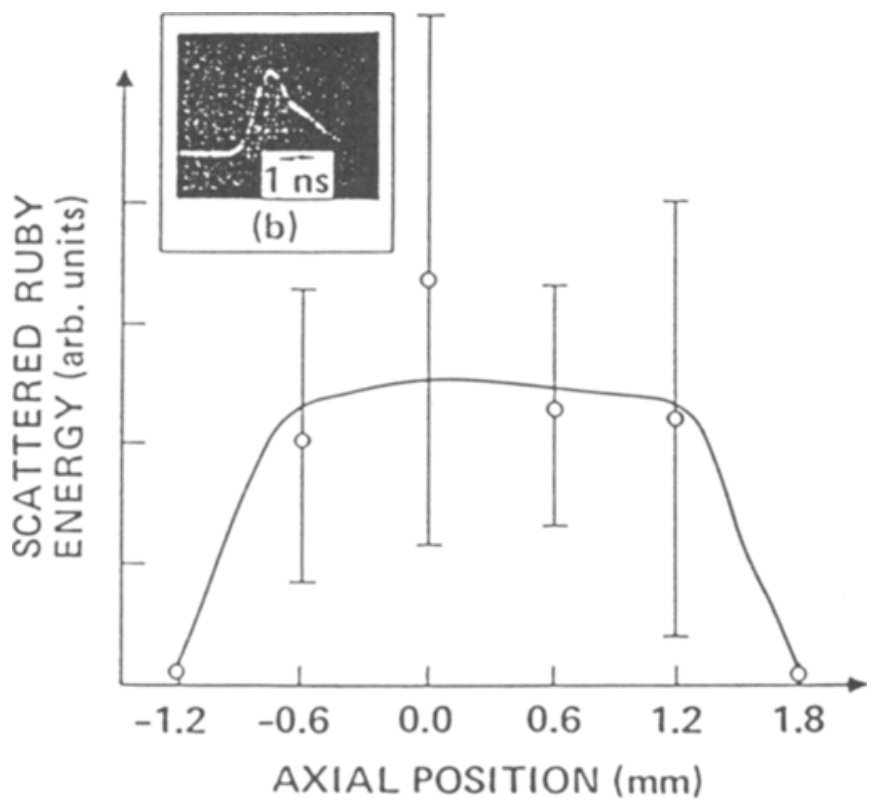

(a)

Fig. 4 (a) Axial profile of the plasma wave and (b) temporal pulse shape of Stokes radiation. 
The time duration of the fast wave can in principle be determined by streaking the Thomson scattered light using a pico-second streak camera. The scattered light was typically $10^{-10}$ of the total incident ruby power of $40 \mathrm{MW}$, too small to streak at very high speeds. Instead, the time duration of the fast wave was inferred from the duration of the Stokes radiation, emitted in the same direction as the incident $\mathrm{CO} 2$, to be approximately $1 \mathrm{~ns}$ as shown in Fig. 4(b).

The thickness $d$ of the plasma wave in Eq. (3) is the thickness over which the perturbed density $\tilde{n} / \mathrm{n}_{O}$ is constant. The measured transverse intensity profile of the $\mathrm{CO}_{2}$ laser in the focal plane was approximately Gaussian with a half-energy spot size of $240 \mu \mathrm{m}$. Since the growth rate of the plasma wave is proportional to the product of the electric fields of the two laser beams, the plasma wave will also have a transverse profile that is approximately Gaussian with the same half width. An equivalent width d (over which the perturbed density is constant) is, therefore, approximately half the spot diameter or $120 \mu \mathrm{m}$.

Substituting for $\left(\mathrm{P}_{\mathrm{TS}} / \mathrm{P}_{\mathrm{O}}\right),\left(\tau_{\mathrm{f}} / \tau_{\mathrm{f}}\right),\left(\mathrm{L}_{\mathrm{f}} / \mathrm{L}_{\mathrm{O}}\right),\left(\mathrm{d} / \lambda_{\mathrm{i}}\right)$ and $\left(n_{0} / n_{c i}\right)$ we obtain the perturbed density of the fast wave $\tilde{n} / n_{o}$ as between $1-3 \%$. The longitudinal electric field is simply related to the perturbed density through Poisson's equation since the plasma wave is an electrostatic wave,

$$
\Delta \mathrm{k} \quad \mathrm{E}_{\mathrm{L}}=4 \pi \mathrm{eñ} \text {. }
$$

Substitution for $\Delta \mathrm{k}$ and $\tilde{\mathrm{n}}$ in eq. (6) leads to longitudinal electric fields of between $300 \mathrm{MeV} / \mathrm{m}$ and $1 \mathrm{GeV} / \mathrm{m}$. This is the first demonstration of longitudinal fields $\geq 1 \mathrm{GeV} / \mathrm{m}$ by any conventional or collective techniques.

Observation of Stokes and anti-Stokes satellites: A rather unique feature of colinear optical mixing in a plasma is the generation of satellites or electromagnetic sidebands that propagate in the same direction as the two frequency laser beam. One or more satellites each frequency downshifted by $\omega_{p}$ (Stokes) and frequency upshifted by $\omega_{p}$ (anti-Stokes) are generated in one or more ways. If the two initial pump beams are intense enough then they can undergo a cascade of decays $\omega_{0} \pm n \omega_{p}$ and $\omega_{1} \pm n \omega_{p}$ via the stimulated Raman forward scattering instability. Pump depletion of the original laser beams is not a problem since the energy given to the forward scattered satellite is much greater than that to the plasma wave. (The latter can in principle be irreversibly lost.) This is dictated by the Manley-Rowe relation or the law of conservation of wave action which states that

$$
\frac{w_{0}}{\omega_{0}}=\frac{w_{1}}{w_{1}}=\frac{w_{p}}{w_{p}}
$$

where $w=N \hbar \omega$. When $\omega_{1}, \omega_{0} \gg \omega_{p}$, multiple cascades are possible because repeated $k$ matching (actually there is a small $k$ mismatch) 


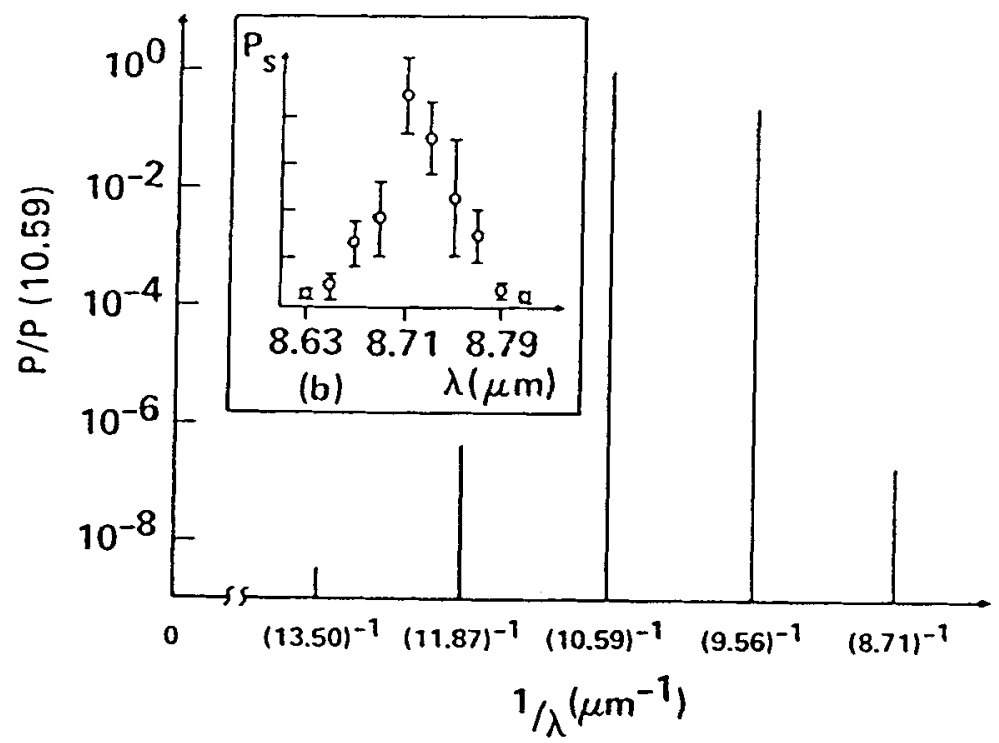

(a)

Fig. 5 (a) Forward scattered IR spectrum showing Stokes and anti-Stokes sidebands and (b) details of the anti-Stokes spectrum.

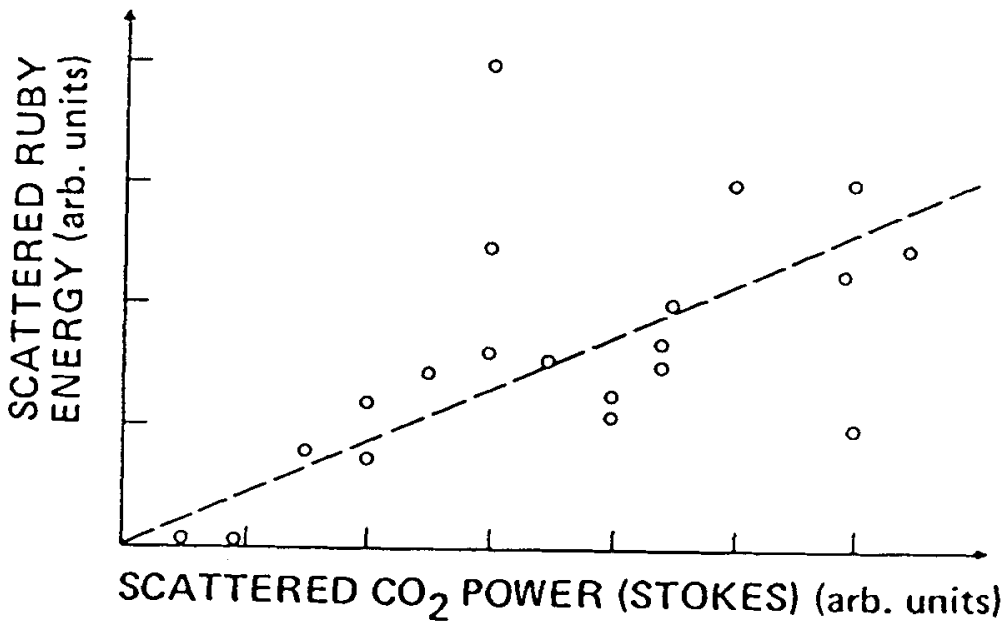

Fig. 6 (a) Correlation between Stokes and ruby Thomson scattered power at $0.4^{\circ}$ (fast wave). 
can occur until the original pump waves have cascaded down to waves with frequencies near $\omega_{p}$.

If the two inital pump waves are not intense enough to produce a spectrum of satellites via the Raman forward instability, then there is the possibility of one or at most two up- and down-shifted satellites being generated by Thomson scattering. The process is analogous to the ruby Thomson scattering discussed earlier, except the pump beams themselves act as probes.

In our experiment the complete satellite spectrum was mapped out by scanning the infrared spectrometer which analyzed the forward scattered radiation. Two frequency downshifted (Stokes) and one frequency upshifted satellites were indeed observed. For the $\mathrm{CO}_{2}$ laser intensities used in our experiment, the threshold scalelength for the Raman forward instability occuring in a parabolic density profile is $6 \mathrm{~mm}$. The fact that the plasma wave is only $1.81 \mathrm{~mm}$ long (a length less than the theshold scalelength) and that the ratio of the Stokes to the incident $10.6 \mu \mathrm{m}$ is only $10^{-6}$ leads us to believe that the Stokes (and similarly anti-stokes) satellite is being generated by Thomson scattering. The most likeIy explanation of the second Stokes satellite which is frequency downshifted by $2 \omega_{p}$ is that it is generated by the incident $10.6 \mu \mathrm{m}$ beam Thomson scattering off the second harmonic of the plasma wave.

As in the case of ruby laser Thomson scattering, the satellites were detected only under two frequency illumination and only when $\omega_{p}$ was close to $\Delta \omega$. As expected, a reasonable correlation was found between the ruby Thomson scattering and the Stokes radiation. This is shown in Fig. 6. Using a Ge:Cu cold detector with a $400 \mathrm{MHz}$ oscilloscope, which have a combined risetime of $600 \mathrm{ps}$, the pulse shapes of the Stokes and the anti-Stokes were resolved. When the oscilloscope risetime is deconvoluted, the satellites were found to have typical times to saturation of $360-530$ ps. This is in excellent agreement with the predictions of the fluid theory.

Competing instabilites: Apart from the SBS and SRS backscatter instabilities a new and rather unexpected effect was discovered via $7.5^{\circ}$ ruby Thomson scattering. Recall that $7.5^{\circ}$ Thomson scattering at $k_{s}=\underline{k}_{i}+\Delta k$ was used to probe for plasma waves generated by the Raman instability and used as a density diagnostic. The $k$ matching assures us that a plasma wave produced by Raman backscatter decay of the incident pump always produces a blue shifted scattered ruby signal. If the plasma wave generated by SRS, which travels in the same direction as the $\mathrm{CO}_{2}$ pump, subsequently decays into another plasma wave travelling in the opposite direction and an ion acoustic wave (this is the so called "parametric-decay instability") the present Thomson scatter geometry can give a red-shifted plasma satellite signal in addition to the blue-shifted plasma satellite. However, even when Raman scatter was large as evidenced by a large blue-shifted satellite, no red-shifted signal was ever detected, When the plasma was irradiated with two frequencies such that $\Delta \omega=$ $\omega_{p}$, the scattered light showed both red- and blue-shifted satellites displaced by $\pm \omega_{p}$ from the SBS signal. The presence of the red and 


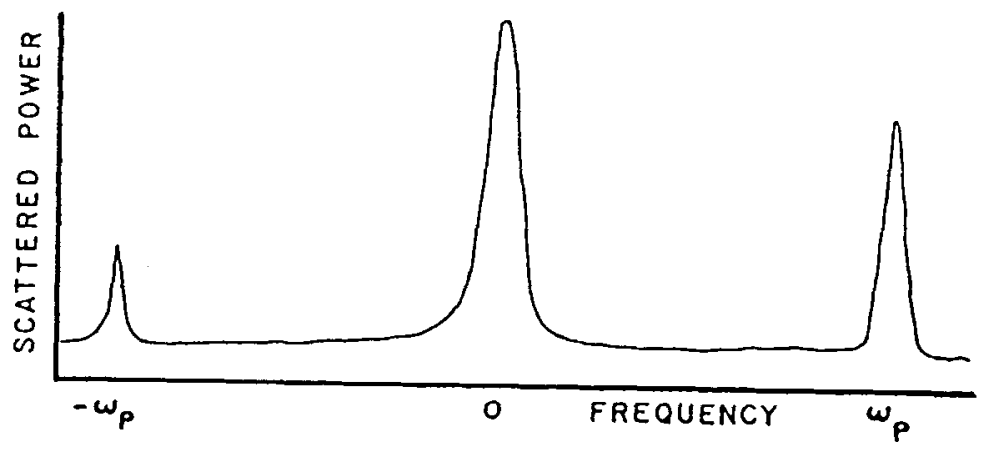

Fig. 7 Result of $7.5^{\circ}$ Thomson scattering at $\omega_{\mathrm{p}}=\Delta \omega$ and under two frequency illumination of plasma.

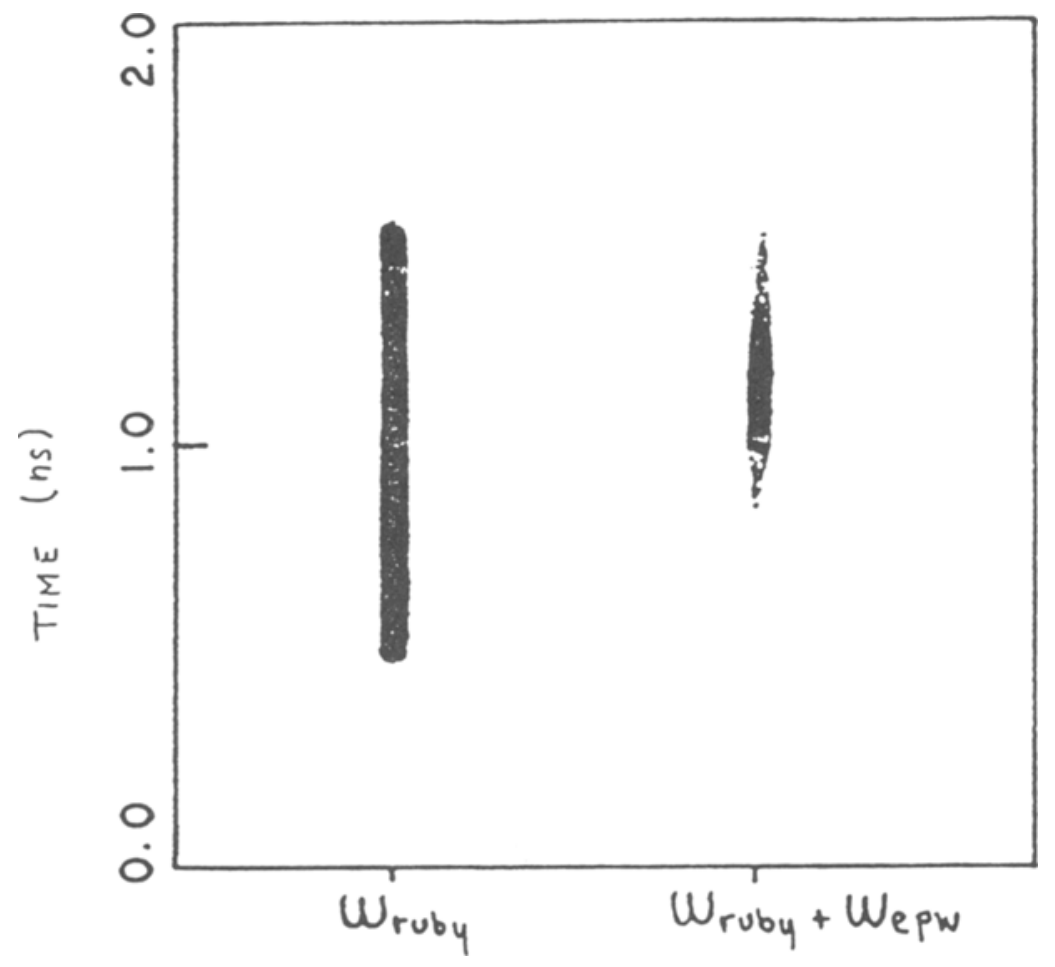

Fig. 8 Time resolved Thomson scattered 1 ight measurement at $7.5^{\circ}$ and $\Delta \omega=\omega_{p}$ showing temporal coincidence of SBS and the plasma wave. 
the blue satellites in Fig. 7 is indicative of two plasma waves propagating opposite one another with frequency $\sim \omega_{p}$ and $k \simeq 2 k_{0}$. The SBS signal and the blue-shifted satellites were time resolved using a streak camera and were found to occur simultanously as shown in Fig. 8. Moreover, whereas single frequency Raman occurs over a range of $\omega_{p}$, the two satellites were a resonant effect in that they were only displaced $\pm \omega_{p}=\Delta \omega$. Another qualitative feature of these satellites was that they seemed to be correlated with SBS. Large SBS produced large satellites.

There are two possible explanations for this rather curious effect. The first and the most likely is the so called induced counter-propagation optical mixing due to SBS. When the plasma is irradiated with two laser lines, SBS scatters a fraction of the energy in each line in the backward direction. The $9.56 \mu \mathrm{m}$ incident line and the SBS induced $10.6 \mu \mathrm{m}$ line beat and excite a slow phase velocity plasma wave propagating in the same direcion as the incident $9.56 \mu \mathrm{m}$ Iine. Similarly, the incident $10.6 \mu \mathrm{m}$ line beats with the SBS $9.56 \mu \mathrm{m}$ 1ine to excite a second slow plasma wave which travels in the opposite direction of the first.

The second and perhaps more intriguing possibility is the so called quasi-resonant mode coupling8. Simulations show that short wavelength ion fluctuations such as those produced by SBS can couple long wavelength, high phase velocity plasma oscillations (such as those produced by colinear optical mixing) to short wavelength, low phase velocity plasma modes which can subsequently be Landau damped.

Further experiments are planned to sort out which of these two (or some other) processes is occuring in the plasma. In any case, SBS plays a crucial role in these experiments, producing very rich wave-wave interaction physics. In any eventual plasma accelerator these competing effects are probably deleterious and must be avoided by going to shorter laser pulses.

\section{CONCLUSIONS}

The observation of the fast wave with the correct frequency, the expected wavenumber and hence, phase velocity, and the rather good agreement of the experimentally observed saturation time and the saturation amplitude with the predicted values from the fluid theory give us confidence that colinear optical mixing in a resonant plasma is a strong effect ${ }^{9}$.

In spite of many competing effects, the desired phenomena, the beat wave, can be unambiguously identified and studied. The laser intensities used in the current experiment are large enough to produce a plasma wave of significant amplitude and, therefore, large longitudinal electric field. Although the beat-wave physics can be studied with nanosecond long laser pulses, much shorter pulses on the order of 50 ps are probably necessary to eliminate the competing instabilities. The current experiments have shown that this collective acceleration scheme is very rich in wavewave interaction physics and future experiments will no doubt 
prove that it is equally rich in wave-particle interaction physics.

\section{ACKNOWLEDGMENTS}

We would like to thank F. F. Chen, J. M. Dawson, T. Katsouleas, W. Mori, D. W. Forslund and J. M. Kindel for many useful discussions regarding this work.

This work was supported by DOE contract DE-AT03-83ER40120,

NSF grant ECS 83-10972 and LLNL University Research Program.

\section{REFERENCES}

1. C. Joshi et a1., Nature 311, 525 (1984).

2. T. Tajima and J. M. Dawson, Phys. Rev. Lett. 43, 267 (1979).

T. Katsouleas and J. M. Dawson, Phys. Rev. Lett. 51, 392 (1983).

3. D. W. Forslund et al., Phys. Rev. Lett. 54, 558 (1984).

4. N. N. Rosenbluth and C. S. Liu, Phys. Rev. Lett. 29, 701 (1972).

5. C. M. Tang et al., App1. Phys. Lett. 45, 375 (1984).

6. C. E. Clayton et al., to be published.

7. C. E. Clayton et al., Phys. Rev. Lett. 51, 1656 (1983).

8. P. K. Kaw et a1., Phys. Fluids 16, 1967 (1973).

9. C. E. Clayton et al., submitted to Phys. Rev. Letters. 